Article

\title{
Effect of Purification Methods on Commercially Available Cellulose Nanocrystal Properties and TEMPO Oxidation
}

\author{
Manali Banerjee ${ }^{1}\left(\mathbb{D}\right.$, Sisira Saraswatula ${ }^{2}$, Anna Williams ${ }^{2}$ and Blair Brettmann ${ }^{1,3, *(D)}$ \\ 1 School of Materials Science and Engineering, Georgia Institute of Technology, Atlanta, GA 30332, USA; \\ bmanalib@gatech.edu \\ 2 Wallace H. Coulter Department of Biomedical Engineering, Georgia Institute of Technology, Atlanta, \\ GA 30332, USA; ssaraswatula3@gatech.edu (S.S.); ansowi16@gmail.com (A.W.) \\ 3 School of Chemical and Biomolecular Engineering, Georgia Institute of Technology, Atlanta, GA 30332, USA \\ * Correspondence: blair.brettmann@chbe.gatech.edu; Tel.: +1-404-894-2535
}

Received: 9 May 2020; Accepted: 13 June 2020; Published: 16 June 2020

\begin{abstract}
Cellulose nanocrystals (CNCs) are attractive for use in polymer composites, biomedical applications, and barrier packaging. In all of these applications they are mixed with other components and compatibility is a major design consideration, as CNCs naturally have a high density of surface hydroxyl groups and primarily disperse well in polar media. Numerous surface modification approaches have been used to address these issues, but challenges remain due to the variability in the commercially available CNC materials. CNCs can be produced from biomass using several extraction methods, most notably acid hydrolysis and biomass extraction, also known as the American Value Added Pulping process. The production method of the CNC material has an impact on both physical and surface properties of CNCs, including size, shape, crystal structure, and zeta potential. In addition, post-treatments can be used to purify the $\mathrm{CNC}$ material and further alter these properties. This work studies the properties of CNCs from three different commercial suppliers and after conducting three different post-treatments: dialysis, Soxhlet extraction, and acetone washing to understand the effect of the commercial source and purification on CNC surface properties and modification via 2,2,6,6-tetramethylpiperidine-1-oxyl radical (TEMPO) mediated oxidation. We show that there is significant variation in CNC physical and surface properties between different commercial suppliers before and after purification. Importantly, we show that for CNCs produced through acid hydrolysis, acetone washing or Soxhlet extraction in ethanol decreases the achievable degree of TEMPO modification, but makes it more consistent between the different commercial suppliers. This has important implications for improving reproducibility in CNC research as well as aiding the expanding fields of applications.
\end{abstract}

Keywords: cellulose; nanocellulose; surface modification; purification; TEMPO oxidation

\section{Introduction}

Cellulosic materials can be extracted from a variety of natural biomass sources including trees, grasses, hemp, and tunicate [1-3]. They show low toxicity [4,5] and their fibers are naturally high in strength and stiffness, making them suitable as reinforcement materials in textiles and composites [6-8] as well as attractive for biomedical and pharmaceutical applications [6,8,9]. Particularly promising forms of cellulose are nano-sized crystals and fibers. A number of researchers have investigated cellulose nanofibers (CNFs) and cellulose nanocrystals (CNCs), taking advantage of the small size for enhancing mechanical properties and the high specific surface area for chemical interactions and modifications. Applications of cellulose nanomaterials range from reinforcing composites $[10,11]$ to 
drug delivery and crystallization systems. [12-15]. Due to the variety of promising applications for cellulose nanomaterials, an understanding of the variability in $\mathrm{CNC}$ properties due to commercial supplier and purification is essential.

A powerful property of $\mathrm{CNCs}$ is the large specific surface area containing a high density of hydroxyl groups, which can be modified through various chemistries. This can be done using surfactants [16,17], molecular grafting [18,19], or polymer grafting [20,21]. Other surface modifications are used to improve gelation in water and organic solvents [14,22]. In many of these cases, the surface reaction begins by converting the hydroxyl groups to more reactive carboxyl groups via 2,2,6,6-tetramethylpiperidine-1-oxyl radical (TEMPO) mediated oxidation [23-25]. The carboxyl groups on oxidized-CNCs (O-CNCs) can be used to form films with barrier properties [26,27], to enable crosslinking in alginate solutions [28] or as a starting point for further modification, such as polymer grafting [23] or carbodiimide coupling [24]. Though this is a crucial surface modification in the CNC field, the effect of CNC commercial supplier and purification method on the extent of TEMPO oxidation has not been previously studied.

Cellulose nanomaterials do not occur at their small size in nature but are instead extracted from bulk sources such as trees, plants, or tunicates [3,29]. They have a hierarchical structure, forming fibers on the largest scale, which are composed of microfibrils made from crystalline and amorphous cellulose regions (Figure 1) [3]. Disruption of the microfibril structure enables the removal of all or parts of the amorphous regions of the material, leading to the nanoscale forms of cellulose, CNFs, and $\mathrm{CNCs}$, with CNCs resulting from removal of a majority of the amorphous regions [3,30]. Breaking the cellulose structure down into smaller components requires harsh extraction methods such as the use of strong acids or enzymatic systems [31-33], which can modify the surface chemistry of the CNCs and $\mathrm{CNFs}$, leading to strong dependencies of material properties on the commercial source and extraction method $[3,34]$. This has been recognized recently in the community and new efforts have emerged to develop benchmarking standards for CNC materials $[35,36]$. The effect of batch-to-batch variation and purification for a single $\mathrm{CNC}$ source on reproducibility of surface modification has also been studied in this context $[37,38]$. Here, we use these principles on a wider variety of commercially available nanocelluloses, including one that is expected to have no surface charge. We use three different purification methods with these CNCs and extend our analysis to understand how TEMPO oxidation of these CNCs is affected by differences in the commercial source and purification method.

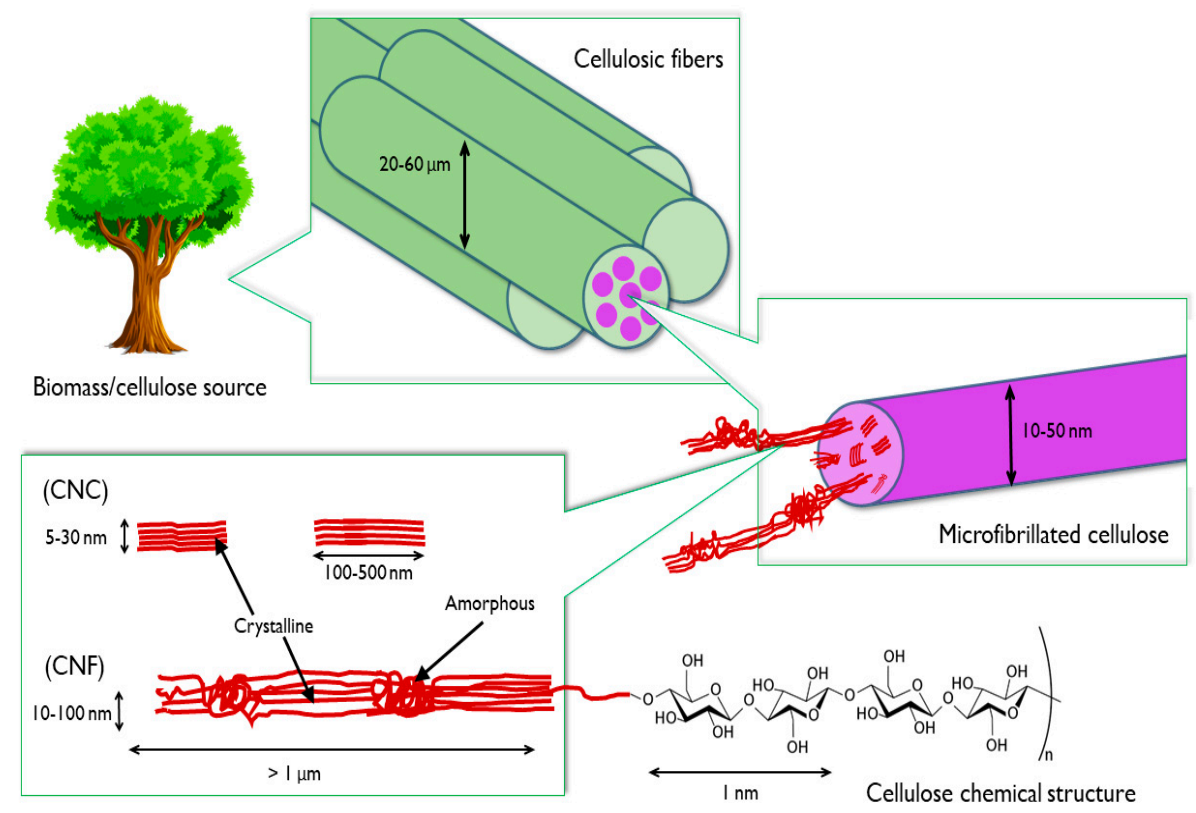

Figure 1. Schematic representation of nanocellulose showing the hierarchical structure. 
Two of the main producers of nanocellulose in North America, the Forest Products Laboratory (FPL) and Celluforce (CF), use sulfuric acid hydrolysis to produce CNCs from wood pulp. After hydrolysis, FPL sells the material as an aqueous dispersion with a $11.8 \mathrm{wt} \%$ solids content, while CF sells the material as a suspension or a spray dried powder. Lately, there has also been the development of a biorefining process by GranBio, which first chemically pretreats biomass with sulfur dioxide $\left(\mathrm{SO}_{2}\right)$ and ethanol to remove lignin and the amorphous parts of the cellulose microfibrils and then mechanically extracts CNCs from the treated biomass $[39,40]$. These preparation methods are extremely important when considering CNCs or CNFs for commercial products, especially in delicate application areas such as healthcare or electronic materials.

Since the commercial CNCs are produced from natural cellulose with minimal clean-up, post-treatments such as dialysis, washing with acetone, and Soxhlet extraction can be used to provide more uniform surface properties [36,37]. Dialysis can be used to remove water soluble salts that may be in the CNC suspension (Figure 2a) [41,42]. Extraction with solvents (Figure 2b) and simple solvent washing (Figure 2c) can be used to dissolve and remove other organic impurities that can alter the properties of the CNC surfaces [36,37]. Impurities may include hemicelluloses, lignin, and various other mono and disaccharides as well as other extractives that are in plants including vanillic acid and plant metabolites. These three post-treatments are expected to modify the CNC particles and their dispersion in water and improve the overall consistency and predictability of the materials, which we will investigate through their effects on the surface modifications of CNCs, specifically TEMPO oxidation, which is a commonly used CNC modification $[24,43,44]$.

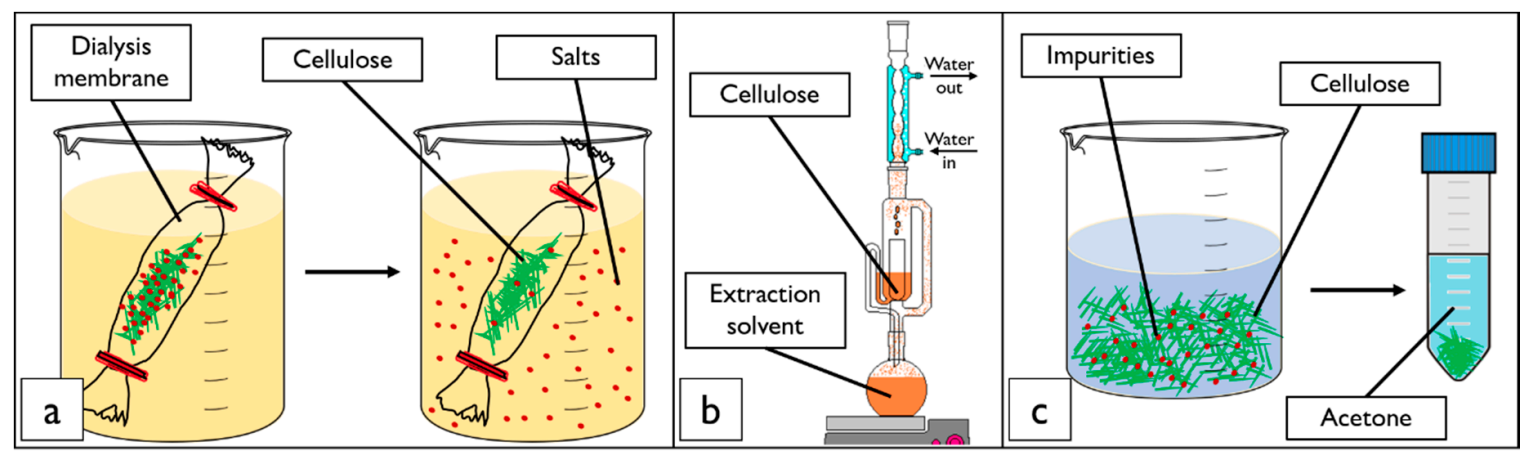

Figure 2. Schematics of post treatments on cellulose nanocrystals (CNCs)—(a) dialysis, (b) Soxhlet extraction in a solvent, and (c) washing with acetone.

In this work, we examine three commercially available batches of CNCs, obtained from FPL, $\mathrm{CF}$, and GranBio and three different purification methods, dialysis, Soxhlet extraction, and acetone washing. We characterize the CNC particle size, crystal structure, and surface charge and perform TEMPO-oxidation on each of the materials. We show how the different post-treatments affect the physical and surface properties of CNCs and use this information to understand the impact of CNC commercial supplier and post-treatment on the extent of surface modification via TEMPO-oxidation.

\section{Materials and Methods}

CNCs were obtained from three different commercial suppliers: an $11.8 \mathrm{wt} \%$ slurry of CNCs in water was produced by the US Department of Agriculture FPL, (Madison, WI, USA-Lot\# 2014-FPL-CNC-064) and distributed by the University of Maine Process Development Center, a spray dried powder was obtained from CF (Windsor, Quebec, Canada), and a slurry of 3.27 wt\% BioPlus ${ }^{\circledR}$ CNC (denoted BP in this work) in water was generously donated by GranBio (Atlanta, USA), which was produced using their American Value Added Pulping (AVAP) process [39]. We used one batch of each supplied material for these studies and did not examine batch-to-batch variation in this work. We use the terminology "cellulose nanocrystal" to describe all three supplied materials for consistency with commercial terminology and clarity in working with these materials, but do not claim that all 
of the supplied materials conform to ISO or CSA standards for CNCs. Both of the acid hydrolyzed CNCs were received in their sodium form. TEMPO was obtained from TCI. Sodium bromide $(\mathrm{NaBr})$ and sodium hypochlorite $(\mathrm{NaOCl})$ were obtained from Alfa Aesar. Ethanol was obtained from Acros Organics. Acetone was obtained from Fisher Chemical. FPL and BP CNCs were used as received or freeze dried in our lab using a FreeZone 2.5 L freeze dryer (Labconco, Kansas, USA). All other materials were used as received. Dialysis membranes were obtained from Ward's Natural Science and were made of regenerated cellulose from cotton linters.

\subsection{Cellulose Nanocrystal Production Methods}

CNC extraction was not performed in our lab as we obtained the pre-made CNCs from the three vendors. Briefly, their production methods are described here. FPL starts with strip-cut dissolving pulp and performs sulfuric acid hydrolysis to obtain CNCs with sulfate ester groups on the surface. The CNCs are then fractionated and concentrated into an aqueous dispersion [31]. CF starts with bleached Kraft pulp and uses a similar sulfuric acid hydrolysis process to obtain CNCs with sulfate ester groups on the surface. After hydrolysis, the CNCs are diluted and neutralized using sodium hydroxide. For the material we used, this is then spray dried and sold as a powder [36,45]. GranBio uses their patented AVAP process to produce BP CNCs. This is done using acidic sulfur dioxide and ethanol to fractionate biomass and remove lignin and the amorphous portions of the cellulosic materials. The CNCs are then mechanically treated to separate the CNC particles. This process leaves the CNC surface charge-free $[39,46]$.

\subsection{Cellulose Nanocrystal Purification Treatments}

For the dialysis purification, aqueous suspensions of CNCs were dialyzed against deionized water. The water-to-suspension volume ratio was 100:1 and, over the course of dialysis, the water was changed five times at a rate of once per day for 5 days.

Prior to Soxhlet extraction, the CNCs were freeze dried. The extraction was then performed on the CNCs $(1 \mathrm{~g})$ with ethanol in a $500 \mathrm{~mL}$ vessel for $48 \mathrm{~h}$. The resulting CNCs were left to dry overnight before using. For the acetone washing, the CNCs were freeze dried and then suspended in acetone and stirred for $10 \mathrm{~min}$. They were then centrifuged for $10 \mathrm{~min}$ at 10,000 RPM using an Eppendorf centrifuge. The supernatant was removed, and the wash and centrifuge steps were repeated three times for each sample. The resulting washed CNCs were left to dry in air overnight before using.

\subsection{Cellulose Nanocrystal Characterization}

Dynamic light scattering (DLS) provides an approximate size of particles by estimating objects as spheres. Even though CNCs are rod-like particles, DLS can provide a good relative indication of the apparent particle size of different CNCs, especially at low CNC concentrations where aggregation is minimized [35]. DLS was used to obtain the approximate particle size of the CNCs. Solutions of $1 \mathrm{wt} \%$ dried CNCs in water were diluted down to $0.001 \mathrm{wt} \%$ and sonicated with a probe sonicator for complete dispersion. These suspensions were measured at $21^{\circ} \mathrm{C}$ on a Zetasizer (Malvern Instruments, Ltd., Worcestershire, UK) using polystyrene cuvettes. Three measurements of each type of CNC were taken with five runs on each sample. Averages and standard deviations were calculated from these 15 values. The error bars represent the standard deviation.

Transmission electron microscopy (TEM) was used to observe the approximate size and shape of the CNCs using a JEOL100 CX-II TEM instrument (JEOL, Akishima, Tokyo, Japan) with an operating voltage of $100 \mathrm{kV}$. Samples were prepared by drop casting $10 \mu \mathrm{L}$ of $0.5 \mathrm{wt} \% \mathrm{CNCs}$ in water onto carbon coated TEM copper grids placed on filter paper. This was followed by dropping $10 \mu \mathrm{L}$ of a $0.1 \%$ uranyl acetate solution to stain the sample; excess liquid was removed by dabbing with filter paper. The sample was allowed to further dry overnight before imaging. Images obtained from TEM were analyzed using ImageJ to measure the size of individual CNCs. A total of 30 crystals from three different images of each CNC type were measured and the average and standard deviation were 
calculated. The aspect ratio of the CNCs were calculated from length versus width of particles and the uncertainty was determined via propagation of error.

X-ray diffraction (XRD) was performed to obtain the crystal structure and percent crystallinity of the CNCs. This was done using a Panalytical X'Pert Pro Alpha-1 XRD (Malvern Panalytical, Malvern, UK) operated at $40 \mathrm{~mA}$. The range of scanned angles was $5^{\circ}$ to $35^{\circ}$ using a $1 / 4^{\circ}$ anti-scatter slit and a 0.04 radian soller slit. These scans were done on the FPL and BP CNCs after freeze drying and on the CF CNCs as received (spray dried powder). Percent crystallinity of the CNCs was determined using two methods. First, the XRD data was analyzed using PeakFit v4.12 software to perform a deconvolution of the amorphous and crystalline peaks. It was assumed that the amorphous content was the only factor contributing to peak broadening, and an amorphous hump at $21.4^{\circ}$ was used as a reference to determine the crystallinity indices [47-49]. The second method used to measure the crystallinity index (CrI) was the peak-height method developed by Segal et al. [49,50] and is given by Equation (1) [50,51]. $\mathrm{I}_{\mathrm{am}}$ is the intensity minimum between the 110 and 200 peaks for cellulose $\mathrm{I}_{\beta}$, which occurs at around $2 \theta=18.7^{\circ}$ and the $I_{200}$ is height of the 200 peak at $2 \theta=22.6^{\circ}$. The intensity was averaged for $2 \theta \pm 0.05^{\circ}$. Standard deviations were also calculated from these values and measured as a percentage of the average. The higher of these values was taken as the uncertainty $[50,52,53]$.

$$
\mathrm{CrI}=\left[1-\left(\mathrm{I}_{\mathrm{am}} / \mathrm{I}_{200}\right)\right] \times 100
$$

Zeta potential measurements of the CNCs were taken using a Zetasizer Nano Z (Malvern Instruments, Ltd., Worcestershire, UK) with a dip-cell setup. The instrument temperature was set to $25^{\circ} \mathrm{C}$. Approximately $800 \mu \mathrm{L}$ of $0.001 \mathrm{wt} \% \mathrm{CNC}$ solutions was measured in optically clear cuvettes. The Smoluchowski equation was used to calculate the zeta potential. Three samples of each type were measured with five runs on each sample. Averages and standard deviations were obtained from these measurements, and the error bars represent the calculated standard deviations for each type of CNC.

\subsection{Surface Modification-TEMPO Mediated Oxidation}

Oxidation of CNCs was done using the procedure by Habibi et al. [44]. Briefly, about $1 \mathrm{~g}$ of freeze dried CNCs was suspended in $150 \mathrm{~mL}$ water and stirred for $10 \mathrm{~min}$. TEMPO $(0.035 \mathrm{~mol} / \mathrm{mol} \mathrm{CNC})$ and $\mathrm{NaBr}(0.5 \mathrm{~mol} / \mathrm{mol} \mathrm{CNC})$ were added and the suspension was further stirred for $10 \mathrm{~min}$. $\mathrm{NaOCl}$ ( $3 \mathrm{~mol} / \mathrm{mol} \mathrm{CNC}$ ) containing $11-15 \%$ active chlorine was added in a dropwise manner to start the oxidation procedure. The $\mathrm{pH}$ of the mixture was kept between 10.5 and 11.5 with the addition of $0.5 \mathrm{M}$ $\mathrm{NaOH}$. The oxidation was deemed complete after $4 \mathrm{~h}$ of stirring when the $\mathrm{pH}$ of the mixture stopped decreasing rapidly. The reaction was terminated by adding $5 \mathrm{~mL}$ ethanol and stirring for another $30 \mathrm{~min}$. The final product was centrifuged to separate the solids and finally, the oxidized nanocellulose (O-CNC) was freeze-dried to obtain a white powder. Three oxidations were performed on each type of $\mathrm{CNC}$ before and after the purifications.

The degree of oxidation was quantitatively measured using acid-base conductometric titrations following the procedure by Araki et al. [23]. Approximately $50 \mathrm{mg}$ of the freeze-dried O-CNC was suspended in $100 \mathrm{~mL}$ water and stirred. A total of $2 \mathrm{~mL}$ of $0.1 \mathrm{M} \mathrm{HCl}$ was added to the suspension to obtain the carboxylic acid form of O-CNC. This was titrated against $0.1 \mathrm{M} \mathrm{NaOH}$ and Equation (2) was used to calculate the amount of carboxyl group per gram of $\mathrm{CNC}$, where $\mathrm{V}_{\text {plateau }}$ is the volume of $\mathrm{NaOH}$ corresponding to the plateau of the conductometric titration curve, which is attributed to neutralizing the $\mathrm{CNC}$ carboxyl groups, $\mathrm{C}_{\mathrm{NaOH}}$ is the $\mathrm{NaOH}$ concentration, and $\mathrm{W}_{\mathrm{CNC}}$ is the weight of $\mathrm{O}-\mathrm{CNCs}$ used for the titration. Titrations were performed on three separate oxidized samples of each type of $\mathrm{CNC}$ and averages and standard deviations were calculated.

$$
\text { Degree of oxidation }(\mathrm{mmol} / \mathrm{g})=\left(\mathrm{V}_{\text {plateau }} \times \mathrm{C}_{\mathrm{NaOH}}\right) / \mathrm{W}_{\mathrm{CNC}}
$$




\section{Results and Discussion}

\subsection{Physical Properties of as Received CNCs}

CNCs were characterized to determine the physical properties, including size, shape, and crystallinity, of the materials. For the as received material, the average "apparent particle size" as measured via DLS was $125 \pm 6 \mathrm{~nm}$ for the FPL CNCs, $92 \pm 6 \mathrm{~nm}$ for the CF CNCs, and $152 \pm 38 \mathrm{~nm}$ for the BP CNCs (Figure 3, blue bars). The variation in the diameter of FPL and CF CNCs was low, indicating narrow distribution of CNC sizes and low aggregation, which is attributed to the colloidal stability of CNCs produced by sulfuric acid hydrolysis. This method of CNC preparation leaves sulfate half ester groups on the surface of the CNCs, which are charged at neutral $\mathrm{pH}$ and provide colloidal stability [54-56]. The DLS measurements for BP CNCs showed a much wider distribution of particle sizes as seen from the high standard deviation. Examination of the particle size distribution indicated the existence of a large number of aggregates with around $50-60 \%$ of each sample having a diameter of $400 \mathrm{~nm}$ or higher. This higher level of aggregation is due to the lack of sulfate ester groups on the BP particles, which are produced without acid hydrolysis, and subsequent low surface charge and low colloidal stability in water $[40,46]$.

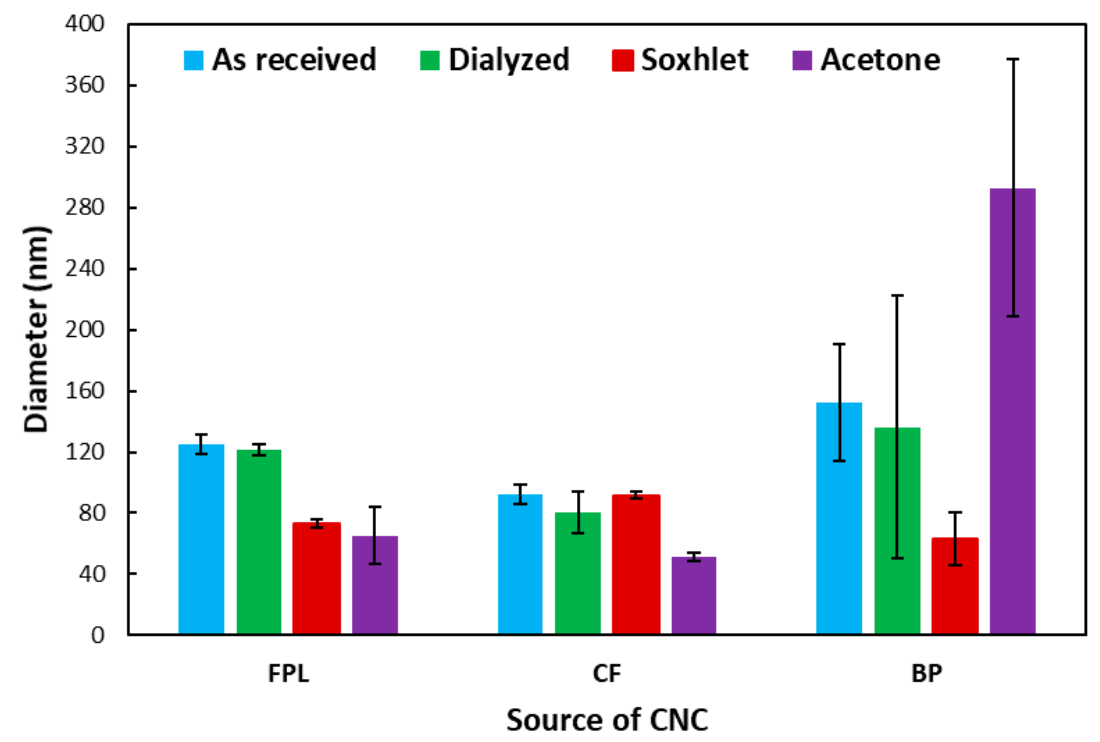

Figure 3. Diameter of all CNCs from dynamic light scattering (DLS) measurements indicating size as received and after post-treatments. Blue bars are for as received, green bars are for dialyzed post-treatment, red bars are for Soxhlet extraction post-treatment, and purple bars are for acetone wash post-treatment.

To complement the DLS results, which treat the particles as spheres, the size and shape of CNCs were observed using TEM (Figure 4). The average lengths of the CNCs were $133 \mathrm{~nm} \pm 34 \mathrm{~nm}$, $79 \pm 30 \mathrm{~nm}$, and $194 \pm 32 \mathrm{~nm}$ for those from FPL, CF, and BP, respectively. Overall, these size values agree with the pattern seen from the DLS results, indicating that the sizes obtained from DLS are reasonable estimates of the approximate CNC whisker sizes and their trends. The sizes obtained from TEM are larger than for DLS for BP, probably due to poorer dispersion in the $0.5 \mathrm{wt} \%$ solution used for TEM imaging, indicating that some of the particles in the TEM images are probably aggregates. Based on the CNC shape, it was determined that the aspect ratio of the BP-CNCs were the highest at $34 \pm 7$ followed by FPL-CNCs at $18 \pm 5$ and CF-CNCs at $8 \pm 3$. 


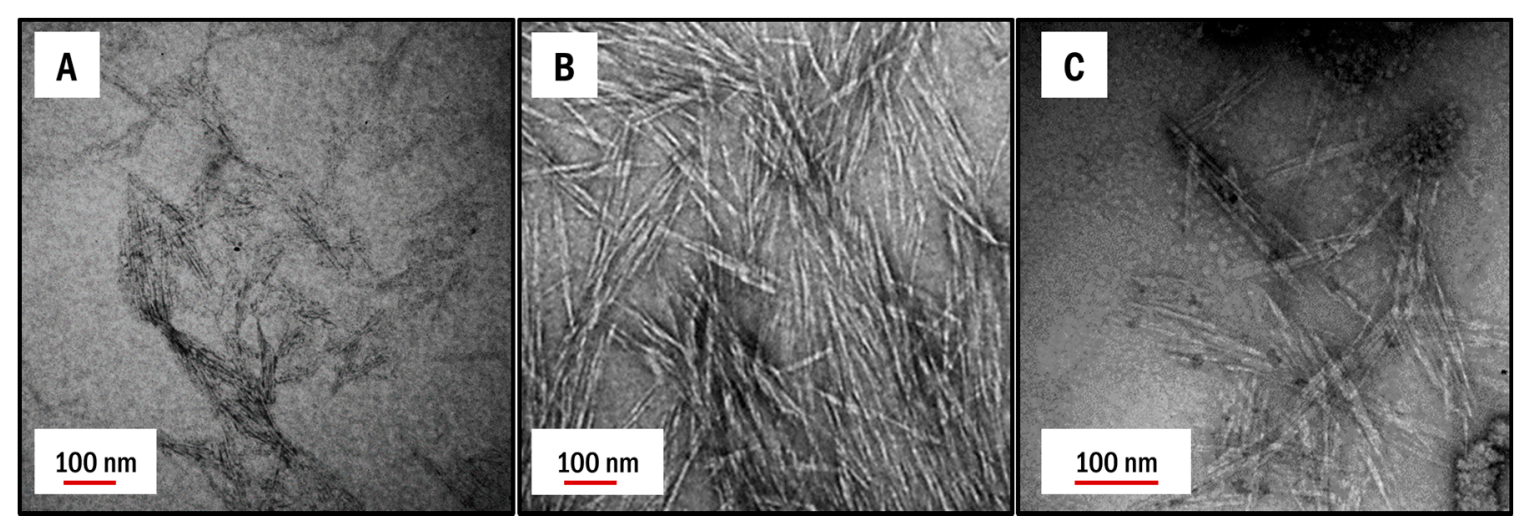

Figure 4. Transmission electron microscopy (TEM) images of as received CNCs: (a) Forest Products Laboratory (FPL), (b) Celluforce (CF), and (c) GranBio BioPlus (BP).

The crystal structure and percent crystallinity of CNCs from the three different commercial suppliers were determined using XRD analysis (Figure 5). It was observed that CNCs from BP and CF contained only cellulose $\mathrm{I}_{\beta}$ crystals, which have a monoclinic crystal structure. Peaks for BP CNCs were observed at $15.8,22.8$, and $34.7^{\circ}$ corresponding to known crystal planes for cellulose $\mathrm{I}_{\beta}$. CNCs from CF show these peaks and an additional peak at 20.7, which corresponds to the 102 plane [52,57]. In addition to peaks at 15.2, 17, and 22.8, FPL CNCs presented with peaks at 12.2, 19.9, and 21.9. The first set are indicative of the presence of the monoclinic crystal structure of cellulose $\mathrm{I}_{\beta}$ and the second set is indicative of cellulose II peaks $[3,36,58]$. The crystallinity of the material determined using PeakFit was based on an underlying amorphous hump at around $21.4^{\circ}$ (not seen in the figure). This method showed that FPL CNCs had the highest total crystallinity at $97 \%$, CF CNCs were $87 \%$ crystalline and BP CNCs were found to have the lowest crystallinity at $68 \%$. The Segal method, which is a good indication of relative crystallinity, gave a CrI of $83.9 \% \pm 5 \%$ for FPL CNCs, $76.8 \% \pm 3 \%$ for CF CNCs, and $75.2 \% \pm 1 \%$ for BP CNCs. Unfortunately, this method does not account for all of the cellulose II present in FPL CNCs since the Segal method needs to be modified to use $\mathrm{I}_{\mathrm{am}}$ at $2 \theta=16^{\circ}[49,50]$; however, this minimum cannot be separated from the $15.2^{\circ}$ and $17^{\circ}$ peaks from the cellulose $\mathrm{I}_{\beta}$ present in the sample. As such, the crystallinity of the FPL CNCs is probably higher than found by this method. While the two methods provide different values of CrI, the relative trend is the same FPL CNC > CF $\mathrm{CNC} \approx \mathrm{BP} C \mathrm{CNC}$. The Segal method was intended as a relative measurement so these variations are understandable [49,50]. The lower crystallinity of the BP CNCs could arise from some of the cellulose nanocrystals being damaged during the mechanical extraction process used by GranBio; however, since we have limited knowledge of their process, it is hard to conclude this definitively.

The zeta potential of the CNCs was also measured (Figure 6, blue symbols). Zeta potential is a measure of the electrical potential at a solid/liquid interface, and it is a function of the surface charge and surrounding suspension medium. We use it here as an indicator of the relative particle surface charge as we maintain a consistent suspension medium (DI water), but it is not a specific measure of the surface charge. Based on typical characterizations of zeta potential, we consider all zeta potential values lower than $-30 \mathrm{mV}$ to be strongly negatively charged (red zone), zeta potential values between -15 and $-30 \mathrm{mV}$ to be moderately negatively charged (yellow zone), and zeta potential values between 0 and $-15 \mathrm{mV}$ to be low to no surface charge (blue zone). We compare samples by zone due to high variation in specific zeta potential values, particularly for non-spherical particles such as CNCs [59]. It was observed that both CF and FPL had similar, highly charged surfaces with zeta potential values of $-37 \pm 3 \mathrm{mV}$ and $-39 \pm 3 \mathrm{mV}$, respectively. BP, obtained from the AVAP process of GranBio, presented with a much lower zeta potential value of $-12 \pm 1 \mathrm{mV}$, indicating low to no surface charge, as expected from that process. 


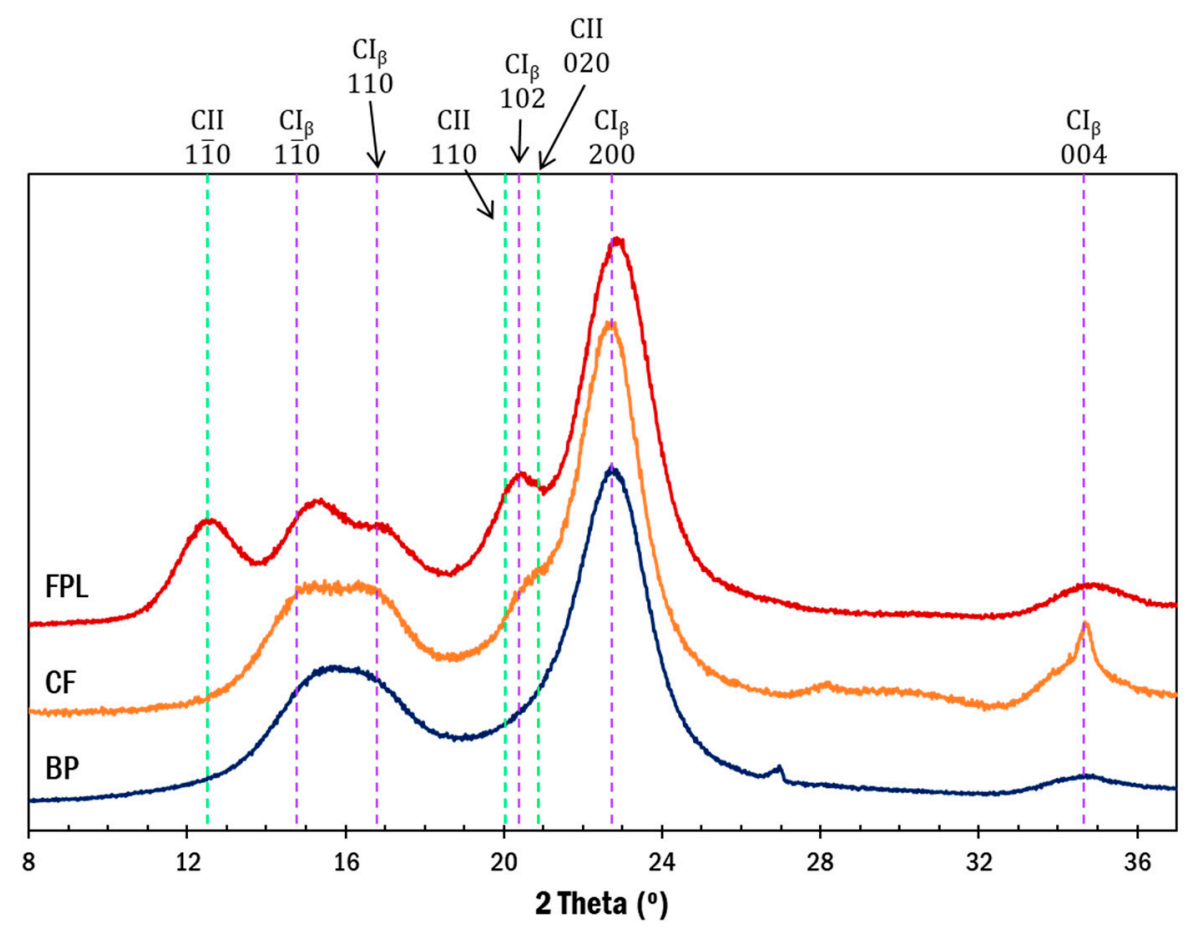

Figure 5. X-ray diffraction (XRD) powder patterns from the three different sources indicating cellulose $\mathrm{I}_{\beta}$ for CF and BP CNCs and cellulose $\mathrm{I}_{\beta}$ and cellulose II for FPL CNCs. Miller indices of the peaks are shown on the graph $\left(\mathrm{CI}_{\beta}=\right.$ Cellulose $\mathrm{I}_{\beta}$-purple lines; $\mathrm{CII}=$ Cellulose $\mathrm{II}$-green lines $)$.

\section{Source of CNC}

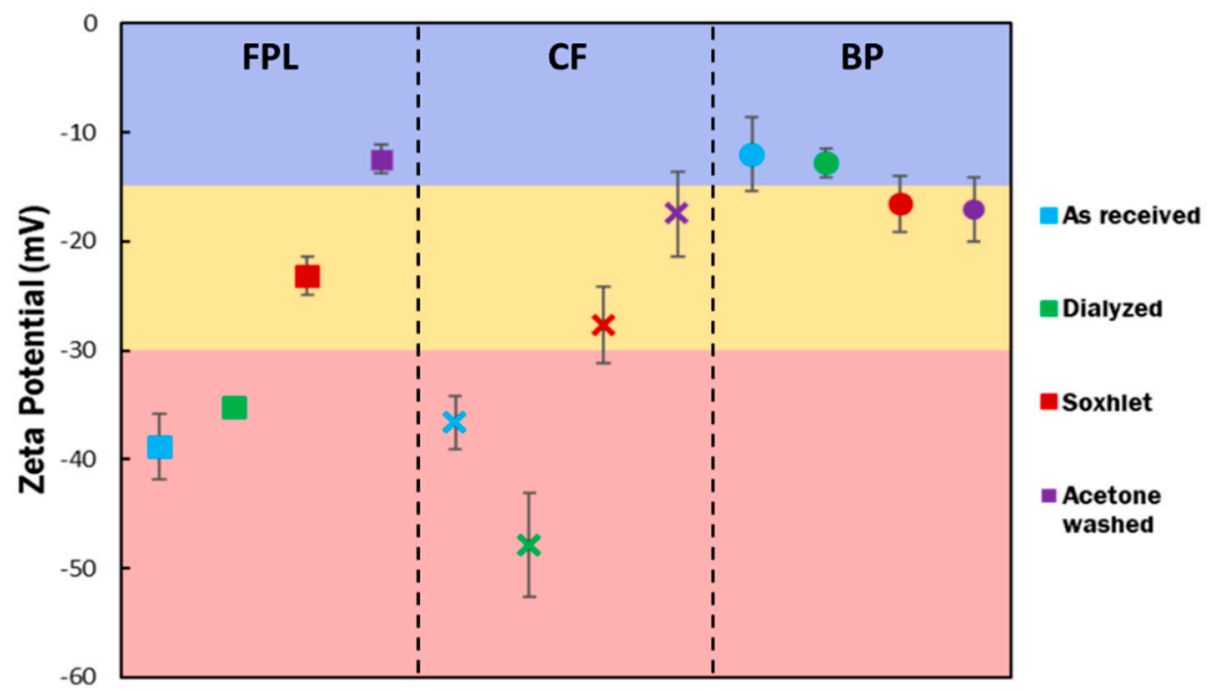

Figure 6. Zeta potential values of CNCs (squares are FPL, crosses are $\mathrm{CF}$, and circles are BP), as received and after post-treatments (blue is as received, green is dialyzed, red is Soxhlet extracted, and purple is acetone washed). Regions are colored to highlight amount of charge- - blue is low to no charge, yellow is moderately charged, and red is highly charged.

\subsection{Physical Properties of Purified CNCs}

After examining the as received CNCs, we performed three different post-treatments on the materials in order to observe how purifying the different as received $\mathrm{CNC}$ materials affected their physical and surface properties. The average size of each of the different CNCs did not significantly change after 5 days of dialysis (Figure 3, green bars). The dialyzed CNC DLS measurements were within error of the as received material. Dialysis removes residual salts and free acid molecules that 
might have been left over from the hydrolysis steps. These salts would cause minor particle aggregation by reducing surface charge [60] or can be present near the CNC particles, which would increase the observed diameter from DLS measurements; however, these results show that this was not significant for these materials and the dialysis purification treatment did not have an effect on CNC sizes.

Soxhlet extraction with ethanol was performed for $48 \mathrm{~h}$ on freeze dried samples of CNCs in order to remove impurities present on the surface. It has been noted previously that extraction with ethanol is able to remove xylobiose, 1,6-anhydroglucose, vanillic acid, and 3,4,5-trimethoxyphenol from the surface of CNCs obtained from bleached cotton [19,37]. This results from the slightly alkaline nature of ethanol, which can be used to hydrolyze, dissolve, and remove the impurities on CNCs [61]. A significantly large reduction in the size of CNCs was seen after this purification treatment (Figure 3 , red bars). The diameter of FPL CNCs after Soxhlet extraction with ethanol was reduced to $73 \pm 2 \mathrm{~nm}$ as compared to $125 \pm 6 \mathrm{~nm}$ for the as received material. This could indicate that extraction with a polar solvent may enable the removal of some amount of impurities from the surface, which could decrease the particle size directly or improve dispersion by removing impurities that cause attractive interactions between particles.

CNC samples were also washed with acetone and a large decrease in the average diameter of the CNCs was observed after the acetone wash treatment (Figure 3, purple bars). For acetone washed FPL CNCs, the diameter was $65 \pm 18 \mathrm{~nm}$ as compared to $125 \pm 6 \mathrm{~nm}$ for the as received material. This indicates that a large amount of organic material on the CNC surface was removed by dissolving in acetone, which is a good solvent for many organic impurities. Again, removal of impurities may decrease the particle size directly or reduce aggregation of the particles, leading to lower measured apparent particle sizes.

The zeta potential of each type of CNC remained approximately the same before and after dialysis (Figure 6, green symbols). The zeta potential for dialyzed FPL CNCs was $-36 \pm 1 \mathrm{mV}$ as compared to the $-37 \pm 3 \mathrm{mV}$ for as received, for dialyzed CF CNCs was $-47 \pm 5 \mathrm{mV}$ as compared to $-39 \pm 3 \mathrm{mV}$ for as received, and for dialyzed BP CNCs was $-13 \pm 1 \mathrm{mV}$ as compared to $-12 \pm 3 \mathrm{mV}$ for as received. These results indicate that dialysis does not have a strong effect on the surface charge, likely due to most of the charged impurities being hydrogen bonded with the $\mathrm{CNC}$ surfaces and not being removed from the surface through this purification method.

After Soxhlet extraction (Figure 6, red symbols), the FPL CNCs showed a reduced zeta potential value of $-23 \pm 2 \mathrm{mV}$ as compared to the $-39 \pm 3 \mathrm{mV}$ from the as received material and the CF CNCs also showed a smaller decrease in zeta potential value from $-37 \pm 2.5$ to $-28 \pm 3.5 \mathrm{mV}$. This indicates that removing some impurities by Soxhlet extraction also removed some charged species that were adsorbed onto the surface of the CNCs. For the BP CNCs, extraction does not significantly change the surface charge properties, and the zeta potential value after extraction is $-17 \pm 2.5 \mathrm{mV}$, which is within error of the as received material at $-12 \pm 3.5 \mathrm{mV}$. The lack of effect from ethanol extraction for Soxhlet extracted BP CNCs can be attributed to the fact that the BP CNCs are produced using ethanol as the solvent to dissolve the biomass. The material has therefore already been altered by ethanol and additional ethanol does not strongly affect it.

After acetone washing, both FPL CNCs and CF CNCs showed a strong decrease in the zeta potential (Figure 6, purple), with acetone washed FPL CNCs having a zeta potential value of $-13 \pm 1 \mathrm{mV}$, and acetone washed CF CNCs having a zeta potential value of $-18 \pm 4 \mathrm{mV}$, leaving the CF CNCs and FPL CNCs slightly charged after the washing. This indicates that a significant amount of organic impurities was dissolved and removed by acetone, which would account for the reduction in the zeta potential. Similar to the extraction process, acetone washing does not significantly alter the BP CNC surface charges. The zeta potential value for acetone washed BP CNCs was $-17 \pm 3 \mathrm{mV}$, which is within error of the as received material. 


\subsection{Surface Modification of CNCS}

The degree to which the CNCs could be oxidized via a TEMPO catalyst mediated oxidation was evaluated at $4 \mathrm{~h}$, which is a typical amount of time used for TEMPO oxidation and leads to mostly complete oxidation [44]. The indication that CNCs were fully oxidized was taken as when the $\mathrm{pH}$ of the oxidation reaction stopped dropping [44,62]. The oxidized CNCs were then characterized using acid-base conductometric titrations to determine the degree of oxidation, reported in $\mathrm{mmol} / \mathrm{g}$ of CNC. This is a common method of determining carboxyl content for oxidized CNCs and measures the conductivity of a solution as a titrant is added. A plateau in the titration curve corresponds with the amount of carboxyl groups $[13,28,62]$.

When performing a TEMPO oxidation for $4 \mathrm{~h}$ and keeping all oxidation conditions constant, with a 3-molar oxidant to CNC ratio, it was observed that FPL CNCs were oxidized to $1.54 \mathrm{mmol} / \mathrm{g}$, CF $\mathrm{CNCs}$ were oxidized to $1.36 \mathrm{mmol} / \mathrm{g}$, and BP CNCs were only oxidized to $1.05 \mathrm{mmol} / \mathrm{g}$. These are typical values found for cellulose nanomaterials produced from wood pulp [63,64].

Each of the post-treated CNCs was also subjected to TEMPO oxidation (Table 1) and the effect of purification on the amount of oxidation possible was measured. Dialysis minimally decreased the degree of oxidation for all of the CNCs, which is expected given the particle size and surface charge results, since removing inorganic residues or salts does not significantly change a material's ability to react chemically and oxidize. Soxhlet extraction, however, decreased the degree of oxidation reached for FPL and CF CNCs. This is surprising, as one might expect the removal of impurities to improve surface modification for typical materials. However, these results indicate that some of the oxidation that was observed on the as received material could be from oxidation of the impurities rather than the cellulose molecules. Additionally, it has been suggested previously that there could be charged oligosaccharides and carboxylic acid impurities adsorbed onto the surface of the CNCs, which are removable by Soxhlet extraction [36,37]. These impurities would artificially increase the degree of oxidation observed and their removal, thus, lowers the degree of oxidation calculated. Similarly, for acetone washing, a decrease in the degree of oxidation for FPL and CF CNCs was observed. Acetone would also be successful in dissolving inorganic impurities on the surface of the CNCs, which could be driving the degree of oxidation higher. As such, washing in acetone would remove these impurities and produce a lower degree of oxidation for just the $\mathrm{CNC}$ material. This purification treatment leads to a greater uniformity of the degree of surface modification across commercial sources, with the degree of oxidation of as received FPL and CF CNCs being $48 \%$ and $27 \%$ higher than BP CNCs, respectively, but only being $14 \%$ higher after acetone washing.

Table 1. Degree of oxidation for $\mathrm{CNCs}$ (using 3 moles of $\mathrm{NaOCl}$ oxidant per mole of cellulose) before and after post-treatments.

\begin{tabular}{cccc}
\hline Post Treatment & FPL (mmol/g) & CF $(\mathbf{m m o l} / \mathbf{g})$ & BP \\
\hline None & $1.54 \pm 0.10$ & $1.36 \pm 0.08$ & $1.04 \pm 0.07$ \\
Dialysis & $1.47 \pm 0.10$ & $1.24 \pm 0.02$ & $0.98 \pm 0.06$ \\
Soxhlet extraction & $1.28 \pm 0.07$ & $1.09 \pm 0.01$ & $1.01 \pm 0.04$ \\
Acetone washing & $1.14 \pm 0.13$ & $1.14 \pm 0.01$ & $1.03 \pm 0.07$ \\
\hline
\end{tabular}

A lower amount of inherent impurities on the surface of BP CNCs would therefore also account for the lower initial degree of oxidation achieved by BP CNCs. Additionally, after purification using Soxhlet extraction and acetone washing, the degree of oxidation achieved by BP CNCs remained the same as the original material, indicating a lower amount of impurities on the surface that could be removed by the solvents. This supports the results obtained using zeta potential measurements shown in Figure 6. The results in Table 1 show that as received CNCs obtained from acid hydrolysis (FPL and $\mathrm{CF}$ CNCs) have a much higher degree of oxidation than as received BP CNCs; however, this is likely due to the presence of surface impurities, which inflate the degree of oxidation values. After washing 
with acetone or Soxhlet extraction with ethanol, FPL-CNCs show the highest degree of oxidation indicating the highest amount of surface modification possible.

\section{Conclusions}

In this work, we studied the physical and surface properties of CNCs from three different commercial suppliers before and after three different post-treatments: dialysis, Soxhlet extraction, and acetone washing. We studied the effect of the CNC supplier and post-treatment on surface modifications of CNC using the degree of substitution possible via TEMPO oxidation as a model reaction. We found that dialysis does not significantly impact the properties of CNC materials nor affect the surface modification abilities of CNCs regardless of the source. The degree of oxidation before and after dialysis remained the same for each type of CNC. Soxhlet extraction had a small effect on the physical properties, especially the particle size, of acid hydrolyzed CNCs produced by FPL and $\mathrm{CF}$, indicating the removal of a few surface impurities, but not on the BP CNCs produced via GranBio's AVAP process. Washing CNCs with acetone produced the largest impact on the physical and surface properties of the CNCs. The sizes of FPL and CF CNCs were significantly reduced, as were the measured zeta potentials of the particles. The degree of oxidation for FPL and CF were also significantly lower, indicating that surface impurities may have been inflating the degree of oxidation calculated for the untreated CNCs, or the impurities themselves were being oxidized. Overall, it is clear from this work that there is significant variation in $\mathrm{CNC}$ physical and surface properties for different commercial suppliers, but that the surface charges and achievable degree of oxidation can be made more uniform across sources by using Soxhlet extraction in ethanol or washing with acetone prior to modification. This has important implications for transitioning CNCs to commercial products for a wide variety of applications where surface modifications, particularly those that rely on a TEMPO-oxidation step, are essential.

Author Contributions: Conceptualization, M.B. and B.B.; methodology, M.B.; validation, M.B., S.S. and A.W.; formal analysis, M.B.; investigation, M.B., S.S. and A.W.; resources, M.B. and B.B.; writing (original draft preparation), M.B.; writing-review and editing, M.B. and B.B.; visualization, M.B. and B.B.; supervision, B.B.; funding acquisition, B.B. All authors have read and agreed to the published version of the manuscript.

Funding: This research received no external funding.

Acknowledgments: M.B. was supported by the Georgia Tech Renewable Bioproducts Institute Paper Science and Engineering Fellowship and S.S. was supported by the Presidents Undergraduate Research Award at Georgia Tech. This work was performed in part at the Georgia Tech Institute for Electronics and Nanotechnology, a member of the National Nanotechnology Coordinated Infrastructure, which is supported by the National Science Foundation (Grant ECCS-1542174). The authors also thank Dong Qin and Ingeborg Schmidt-Krey's lab at Georgia Tech for use of equipment and Nasreen Khan and Nadia Zaragoza for assistance with zeta potential measurements.

Conflicts of Interest: The authors declare no conflict of interest.

\section{References}

1. Wang, Y.; Wei, X.; Li, J.; Wang, F.; Wang, Q.; Kong, L. Homogeneous isolation of nanocellulose from cotton cellulose by high pressure homogenization. J. Mater. Sci. Chem. Eng. 2013, 1, 49-52. [CrossRef]

2. Brinchi, L.; Cotana, F.; Fortunati, E.; Kenny, J.M. Production of nanocrystalline cellulose from lignocellulosic biomass: Technology and applications. Carbohydr. Polym. 2013, 94, 154-169. [CrossRef]

3. Moon, R.J.; Martini, A.; Nairn, J.; Simonsen, J.; Youngblood, J. Cellulose nanomaterials review: Structure, properties and nanocomposites. Chem. Soc. Rev. 2011, 40, 3941-3994. [CrossRef]

4. Vartiainen, J.; Pöhler, T.; Sirola, K.; Pylkkänen, L.; Alenius, H.; Hokkinen, J.; Tapper, U.; Lahtinen, P.; Kapanen, A.; Putkisto, K.; et al. Health and environmental safety aspects of friction grinding and spray drying of microfibrillated cellulose. Cellulose 2011, 18, 775-786. [CrossRef]

5. Kovacs, T.; Naish, V.; O'Connor, B.; Blaise, C.; Gagné, F.; Hall, L.; Trudeau, V.; Martel, P. An ecotoxicological characterization of nanocrystalline cellulose (NCC). Nanotoxicology 2010, 4, 255-270. [CrossRef]

6. Kalia, S.; Dufresne, A.; Cherian, B.M.; Kaith, B.S.; Avérous, L.; Njuguna, J.; Nassiopoulos, E. Cellulose-Based Bio- and Nanocomposites: A Review. Int. J. Polym. Sci. 2011, 2011, 1-35. [CrossRef] 
7. Dufresne, A. Nanocellulose: A new ageless bionanomaterial. Mater. Today 2013, 16, 220-227. [CrossRef]

8. Czaja, W.; Krystynowicz, A.; Bielecki, S.; Brown, R.M.; Huang, L.; Chen, X.; Nguyen, T.X.; Tang, H.; Zhang, L.; Yang, G.; et al. Microbial cellulose-The natural power to heal wounds. Angew. Chem. Int. Ed. 2015, 33, 1-10. [CrossRef]

9. Domingues, R.M.A.; Silva, M.; Gershovich, P.; Betta, S.; Babo, P.; Caridade, S.G.; Mano, J.F.; Motta, A.; Reis, R.L.; Gomes, M.E. Development of injectable hyaluronic acid/cellulose nanocrystals bionanocomposite hydrogels for tissue engineering applications. Bioconjug. Chem. 2015, 26, 1571-1581. [CrossRef]

10. Irvin, C.W.; Satam, C.C.; Carson Meredith, J.; Shofner, M.L. Mechanical reinforcement and thermal properties of PVA tricomponent nanocomposites with chitin nanofibers and cellulose nanocrystals. Compos. Part A Appl. Sci. Manuf. 2019, 116, 147-157. [CrossRef]

11. Muiruri, J.K.; Liu, S.; Teo, W.S.; Kong, J.; He, C. Highly biodegradable and tough polylactic acid-cellulose nanocrystal composite. ACS Sustain. Chem. Eng. 2017, 5, 3929-3937. [CrossRef]

12. Plackett, D.V.; Letchford, K.; Jackson, J.K.; Burt, H.M. A review of nanocellulose as a novel vehicle for drug delivery. Nord. Pulp Pap. Res. J. 2014, 29, 105-118. [CrossRef]

13. Johnson, R.K.; Zink-Sharp, A.; Glasser, W.G. Preparation and characterization of hydrophobic derivatives of TEMPO-oxidized nanocelluloses. Cellulose 2011, 18, 1599-1609. [CrossRef]

14. Banerjee, M.; Saraswatula, S.; Willows, L.G.; Woods, H.; Brettmann, B. Pharmaceutical crystallization in surface-modified nanocellulose organogels. J. Mater. Chem. B 2018, 6, 7317-7328. [CrossRef]

15. Ruiz-Palomero, C.; Kennedy, S.R.; Soriano, M.L.; Jones, C.D.; Valcarcel, M.; Steed, J.W.; Valcárcel, M.; Steed, J.W. Pharmaceutical crystallization with nanocellulose organogels. Chem. Commun. 2016, 52, 7782-7785. [CrossRef]

16. Bonini, C.; Heux, L.; Cavaillé, J.Y.; Lindner, P.; Dewhurst, C.; Terech, P. Rodlike cellulose whiskers coated with surfactant: A small-angle neutron scattering characterization. Langmuir 2002, 18, 3311-3314. [CrossRef]

17. Syverud, K.; Xhanari, K.; Chinga-Carrasco, G.; Yu, Y.; Stenius, P. Films made of cellulose nanofibrils: Surface modification by adsorption of a cationic surfactant and characterization by computer-assisted electron microscopy. J. Nanoparticle Res. 2011, 13, 773-782. [CrossRef]

18. Gousse, C.; Chanzy, H.; Cerrada, M.L.; Fleury, E. Surface silylation of cellulose microfibrils: Preparation and rheological properties. Polymer 2004, 45, 1569-1575. [CrossRef]

19. Eyley, S.; Thielemans, W. Surface modification of cellulose nanocrystals. Nanoscale 2014, 6, 7764-7779. [CrossRef]

20. Lönnberg, H.; Fogelström, L.; Samir, M.A.S.A.; Berglund, L.; Malmström, E.; Hult, A. Surface grafting of microfibrillated cellulose with poly(e-caprolactone)-Synthesis and characterization. Eur. Polym. J. 2008, 44, 2991-2997. [CrossRef]

21. Habibi, Y.; Goffin, A.L.; Schiltz, N.; Duquesne, E.; Dubois, P.; Dufresne, A. Bionanocomposites based on poly( $\varepsilon$-caprolactone)-grafted cellulose nanocrystals by ring-opening polymerization. J. Mater. Chem. 2008, 18, 5002-5010. [CrossRef]

22. Hasani, M.; Cranston, E.D.; Westman, G.; Gray, D.G. Cationic surface functionalization of cellulose nanocrystals. Soft Matter 2008, 4, 2238-2244. [CrossRef]

23. Araki, J.; Wada, M.; Kuga, S. Steric stabilization of a cellulose microcrystal suspension by Poly (ethylene glycol) grafting. Cellulose 2001, 17, 21-27. [CrossRef]

24. Follain, N.; Marais, M.F.; Montanari, S.; Vignon, M.R. Coupling onto surface carboxylated cellulose nanocrystals. Polymer 2010, 51, 5332-5344. [CrossRef]

25. Li, Z.; Renneckar, S.; Barone, J.R. Nanocomposites prepared by in situ enzymatic polymerization of phenol with TEMPO-oxidized nanocellulose. Cellulose 2010, 17, 57-68. [CrossRef]

26. Fukuzumi, H.; Saito, T.; Iwata, T.; Kumamoto, Y.; Isogai, A. Transparent and high gas barrier films of cellulose nanofibers prepared by TEMPO-mediated oxidation. Biomacromolecules 2009, 10, 162-165. [CrossRef]

27. Mendoza, L.; Batchelor, W.; Tabor, R.F.; Garnier, G. Gelation mechanism of cellulose nanofibre gels: A colloids and interfacial perspective. J. Colloid Interface Sci. 2018, 509, 39-46. [CrossRef]

28. Lin, N.; Bruzzese, C.; Dufresne, A. TEMPO-oxidized nanocellulose participating as crosslinking aid for alginate-based sponges. ACS Appl. Mater. Interfaces 2012, 4, 4948-4959. [CrossRef]

29. Mandal, A.; Chakrabarty, D. Isolation of nanocellulose from waste sugarcane bagasse (SCB) and its characterization. Carbohydr. Polym. 2011, 86, 1291-1299. [CrossRef] 
30. Mishra, S.P.; Manent, A.S.; Chabot, B.; Daneault, C. Production of nanocellulose from native cellulose-Various options utilizing ultrasound. BioResources 2012, 7, 422-435.

31. Reiner, R.S.; Rudie, A.W.; Bilodeau, M.A. Process scale-up of cellulose nanocrystal production to $25 \mathrm{~kg}$ per batch at the forest products laboratory. In Production and Applications of Cellulose Nanomaterials; TAPPI: Peachtree Corners, GA, USA, 1998; pp. 21-24.

32. Siró, I.; Plackett, D. Microfibrillated cellulose and new nanocomposite materials: A review. Cellulose 2010, 17, 459-494. [CrossRef]

33. Li, J.; Wei, X.; Wang, Q.; Chen, J.; Chang, G.; Kong, L.; Su, J.; Liu, Y. Homogeneous isolation of nanocellulose from sugarcane bagasse by high pressure homogenization. Carbohydr. Polym. 2012, 90, 1609-1613. [CrossRef]

34. de Souza Lima, M.M.; Borsali, R. Rodlike cellulose microcrystals: Structure, properties, and applications. Macromol. Rapid Commun. 2004, 25, 771-787. [CrossRef]

35. Foster, E.J.; Moon, R.J.; Agarwal, U.P.; Bortner, M.J.; Bras, J.; Camarero-Espinosa, S.; Chan, K.J.; Clift, M.J.D.; Cranston, E.D.; Eichhorn, S.J.; et al. Current characterization methods for cellulose nanomaterials. Chem. Soc. Rev. 2018, 47, 2609-2679. [CrossRef] [PubMed]

36. Reid, M.S.; Villalobos, M.; Cranston, E.D. Benchmarking cellulose nanocrystals: From the laboratory to industrial production. Langmuir 2017, 33, 1583-1598. [CrossRef]

37. Labet, M.; Thielemans, W. Improving the reproducibility of chemical reactions on the surface of cellulose nanocrystals: ROP of e-caprolactone as a case study. Cellulose 2011, 18, 607-617. [CrossRef]

38. Agrawal, A.M.; Dudhedia, M.S.; Zimny, E. Hot melt extrusion: Development of an amorphous solid dispersion for an insoluble drug from mini-scale to clinical scale. AAPS PharmSciTech 2016, 17, 133-147. [CrossRef]

39. Nelson, K.; Retsina, T. Innovative nanocellulose process breaks the cost barrier. TAPPI J. 2014, 13, 19-23. [CrossRef]

40. Kyle, S.; Jessop, Z.M.; Al-Sabah, A.; Hawkins, K.; Lewis, A.; Maffeis, T.; Charbonneau, C.; Gazze, A.; Francis, L.W.; Iakovlev, M.; et al. Characterization of pulp derived nanocellulose hydrogels using AVAP ${ }^{\circledR}$ technology. Carbohydr. Polym. 2018, 198, 270-280. [CrossRef]

41. Johnston, L.J.; Jakubek, Z.J.; Beck, S.; Araki, J.; Cranston, E.D.; Danumah, C.; Fox, D.; Li, H.; Wang, J.; Mester, Z.; et al. Determination of sulfur and sulfate half-ester content in cellulose nanocrystals: An interlaboratory comparison. Metrologia 2018, 55, 872-882. [CrossRef]

42. Abitbol, T.; Kloser, E.; Gray, D.G. Estimation of the surface sulfur content of cellulose nanocrystals prepared by sulfuric acid hydrolysis. Cellulose 2013, 20, 785-794. [CrossRef]

43. Jiang, F.; Hsieh, Y.-L. Self-assembling of TEMPO oxidized cellulose nanofibrils as affected by protonation of surface carboxyls and drying methods. ACS Sustain. Chem. Eng. 2016, 4, 1041-1049. [CrossRef]

44. Habibi, Y.; Chanzy, H.; Vignon, M.R. TEMPO-mediated surface oxidation of cellulose whiskers. Cellulose 2006, 13, 679-687. [CrossRef]

45. Dong, X.M.; Revol, J.F.; Gray, D.G. Effect of microcrystallite preparation conditions on the formation of colloid crystals of cellulose. Cellulose 1998, 5, 19-32. [CrossRef]

46. Nelson, K.; Retsina, T.; Iakovlev, M.; van Heiningen, A.; Deng, Y.; Shatkin, J.A.; Mulyadi, A. American process: Production of low cost nanocellulose for renewable, advanced materials applications. In Springer Series in Materials Science; Springer Nature: Berlin, Germany, 2016; pp. 267-302.

47. Hult, E.L.; Iversen, T.; Sugiyama, J. Characterization of the supermolecular structure of cellulose in wood pulp fibres. Cellulose 2003, 10, 103-110. [CrossRef]

48. Garvey, C.J.; Parker, I.H.; Simon, G.P. On the Interpretation of X-ray diffraction powder patterns in terms of the nanostructure of cellulose I fibres. Macromol. Chem. Phys. 2005, 206, 1568-1575. [CrossRef]

49. Park, S.; Baker, J.O.; Himmel, M.E.; Parilla, P.A.; Johnson, D.K. Cellulose crystallinity index: Measurement techniques and their impact on interpreting cellulase performance. Biotechnol. Biofuels 2010, 3, 10. [CrossRef]

50. Segal, L.; Creely, J.J.; Martin, A.E.; Conrad, C.M. An empirical method for estimating the degree of crystallinity of native cellulose using the X-ray diffractometer. Text. Res. J. 1959, 29, 786-794. [CrossRef]

51. Lee, S.Y.; Mohan, D.J.; Kang, I.A.; Doh, G.H.; Lee, S.; Han, S.O. Nanocellulose reinforced PVA composite films: Effects of acid treatment and filler loading. Fibers Polym. 2009, 10, 77-82. [CrossRef]

52. French, A.D. Idealized powder diffraction patterns for cellulose polymorphs. Cellulose 2014, 21, 885-896. [CrossRef] 
53. Chen, W.; Yu, H.; Liu, Y.; Chen, P.; Zhang, M.; Hai, Y. Individualization of cellulose nanofibers from wood using high-intensity ultrasonication combined with chemical pretreatments. Carbohydr. Polym. 2011, 83, 1804-1811. [CrossRef]

54. Tardy, B.L.; Yokota, S.; Ago, M.; Xiang, W.; Kondo, T.; Bordes, R.; Rojas, O.J. Nanocellulose-surfactant interactions. Curr. Opin. Colloid Interface Sci. 2017, 29, 57-67. [CrossRef]

55. Mascheroni, E.; Rampazzo, R.; Aldo, M.; Piva, G.; Bonetti, S.; Piergiovanni, L. Comparison of cellulose nanocrystals obtained by sulfuric acid hydrolysis and ammonium persulfate, to be used as coating on flexible food-packaging materials. Cellulose 2016, 23, 779-793. [CrossRef]

56. Zhong, L.; Fu, S.; Peng, X.; Zhan, H.; Sun, R. Colloidal stability of negatively charged cellulose nanocrystalline in aqueous systems. Carbohydr. Polym. 2012, 90, 644-649. [CrossRef]

57. Briois, B.; Saito, T.; Petrier, C.; Putaux, J.; Nishiyama, Y.; Heux, L.; Molina-Boisseau, S. I $\alpha$-> I $\beta$ transition of cellulose under ultrasonic radiation. Cellulose 2013, 20, 597-603. [CrossRef]

58. Kafle, K.; Greeson, K.; Lee, C.; Kim, S.H. Cellulose polymorphs and physical properties of cotton fabrics processed with commercial textile mills for mercerization and liquid ammonia treatments. Text. Res. J. 2014, 84, 1692-1699. [CrossRef]

59. Pate, K.T.; Safier, P. Chemical metrology methods for CMP quality. In Advances in Chemical Mechanical Planarization (CMP); Woodhead Publishing: Hillsboro, OR, USA, 2016; pp. 299-325.

60. Fall, A.B.; Lindström, S.B.; Sundman, O.; Ödberg, L.; Wågberg, L. Colloidal stability of aqueous nanofibrillated cellulose dispersions. Langmuir 2011, 27, 11332-11338. [CrossRef]

61. George, J.; Sabapathi, S. Cellulose nanocrystals: Synthesis, functional properties, and applications. Nanotechnol. Sci. Appl. 2015, 8, 45-54. [CrossRef]

62. da Silva Perez, D.; Montanari, S.; Vignon, M.R. TEMPO-mediated oxidation of cellulose III. Biomacromolecules 2003, 4, 1417-1425. [CrossRef] [PubMed]

63. Saito, T.; Kimura, S.; Nishiyama, Y.; Isogai, A. Cellulose nanofibers prepared by TEMPO-mediated oxidation of native cellulose. Biomacromolecules 2007, 8, 2485-2491. [CrossRef] [PubMed]

64. Okita, Y.; Saito, T.; Isogai, A. Entire surface oxidation of various cellulose microfibrils by TEMPO-mediated oxidation. Biomacromolecules 2010, 11, 1696-1700. [CrossRef] [PubMed] 